\title{
Prevalence of left ventricular diastolic dysfunction in OSA patients: a retrospective study
}

\author{
Rantu Paul*, Rana Dey*, Nirjoo Barooah*, Saikat Nag\#, Saurabh \\ Maji**, Parthasarathi Bhattacharyya\# \\ *Clinical research assistant, ** Research fellow, \# Consultant - Institute of Pulmocare and Research, Kolkata

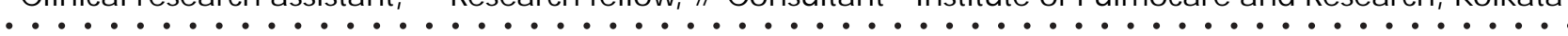 \\ Indian J Sleep Med 2011; 6.2, 50-53
}

\begin{abstract}
Introduction: OSA has been increasingly implicated in the initiation and progression of cardiovascular diseases. We attempted to find the association of LVDD with OSA in terms of its frequency as well as in terms of several important parameters.

Materials and methods: It was a retrospective study where 32 OSA patients diagnosed by polysomnography who also underwent echocardiography were looked for the presence of LVDD. The patients were divided into two groups based on the presence or absence of LVDD and compared them according to different parameters.

Results: The overall prevalence of LVDD in our OSA patients has been found to be $46.8 \%$. There has been found to be no significant differences as per the different parameters considered. On the contrary, severity of sleep apnea was found to be higher in the non LVDD group tallying to their higher BMI, higher neck and waist circumference.

Conclusion: It has not been possible to associate severity of OSA with risk or prevalence of LVDD as there has been found to be no difference between the two groups, with and without LVDD. This area needs further probing so as to look into the origin of LVDD in certain OSA sufferers.

Keywords: OSA (O bstructive Sleep Apnea), LVDD (Left Ventricular Diastolic Dysfunction), CPAP (Continuous Positive Airway Pressure), CO PD (Chronic O bstructive Pulmonary Disease), ESS (Epworth Sleepiness Score)
\end{abstract}

\section{Introduction}

0 bstructive sleep apnea (OSA), being a fairly common, but neglected disorder has been associated with several cardio vascular morbidities as hypertension, acutemyocardial infarction, cardiac arrhythmias, coronary artery disease, heart failure and stroke. ${ }^{1-3}$ Some studies have recorded that

\section{Address for correspondence}

\section{Mr Rantu Paul}

Institute of Pulmocare and Research, CB 16, Sector I, Salt Lake, Kolkata-700 064.

E-mail: ipcr_india@yahoo.com

Indian Journal of Sleep M edicine (IJSM ), Vol. 6, N o. 2, 2011 apparently unexplained left ventricular failure in some patients is actually contributed by OSA. Incidentally, diastolic heart failure has been found to be more common than systolic heart failure. ${ }^{4}$ Such diastolic dysfunction may have important association with OSA severity and it is observed that treatment of OSA with nasal CPAP may actually improve the diastolic dysfunction in OSA. ${ }^{5}$

We looked for association of LVDD with OSA in terms of its frequency and tried to see the difference, if any, between patients with and without LVDD, especially in relation to several different important parameters taken into consideration in evaluation of OSA. 


\section{Materials and Methods}

It has been a retrospective study. We looked at our records for the presence of left ventricular diastolic dysfunction in our patients of OSA since the time we started keeping a note of positive findings of echocardiography (LVDD, LVSD, RM WA, PAH), if done in our patients. For all these patients, we had detailed record of sleep study results done at our lab by EmblaS 4000 machinebetween June2009 and July 2010.

Based on the presence or absence of LVDD, we have divided the patients into two groups and compared them according to the different parameters as-

a. M orphometric measurements (age, BMI, neck circumference, waist circumference)

b. Resting general condition ( $\left.\mathrm{PR}, \mathrm{SaO}_{2}, \mathrm{BP}\right)$

c. Co morbidities (H ypertension, COPD, diabetes, IHD )

d. Addiction habits (smoking and alcoholism)

e. Sleepiness (ESS score) and different sleep related parameters

f. Oxygenation status

All data were presented as mean \pm SD unless otherwise stated. The statistical analysis was performed by unpaired t test.

\section{Results}

We could collect the data of 32 patients with significant OSA who were predominantly men (27 of 32 patients). $M$ ean age of the patients were $50.53 \pm 9.63$ years and the mean BM I was $33.32 \pm 6.11 \mathrm{~kg} / \mathrm{m}^{2}$ respectively and the mean ESS score was $11.93 \pm 6.68$. Polysomnography revealed severe OSA in all, with a mean AH I of $51.84 \pm$ 30.25. $O$ ut of 32 patients screened, LVDD was present in 15 patients and in 17 patients LVDD was found to be absent. The details of these two groups of patients were charted on different parameters as shown in table 1.

The results reflect that there have been no significant differences as per demographic data, presence of co morbidities many of which are known to pre dispose LVDD and different clinical measurements. On comparing the different sleep related parameters as ESS, total sleep duration, sleep efficiency, apnoea or hypopnea per hour, the difference between the two groups were again not found to be significant. Except for duration of snoring, all the other parameters were more severe in patients without LVDD. Tallying to the higher BM I, higher neck and waist circumference, severity of sleep apnea was higher in the non LVD D group.

\section{Discussion}

OSA is a common disorder characterized by periodic reduction or cessation of airflow due to narrowing of the upper airways during sleep. ${ }^{6}$ Prevalence surveys estimate that $2 \%$ of women and $4-5 \%$ of men of middle age are affected by this syndrome. ${ }^{7}$ It has significant deleterious effects on patients' health, as there is considerable evidence suggesting an increased risk for cardiovascular diseases. ${ }^{8}$ H owever, many risk factors for OSA, including male gender, advanced age, and obesity, are the same for cardiovascular disease, which makes it difficult to recognize the role of OSA as an independent risk factor. Some studies have demonstrated an association of OSA with certain cases of left ventricular (LV) failure of otherwise unknown etiology, especially diastolic dysfunction. ${ }^{10-11}$

Left ventricular diastolic dysfunction is a condition with increased resistance to filling of the left ventricle, leading to an inappropriate rise in the diastolic pressurevolume relationship and causing symptoms of pulmonary congestion during exercise. ${ }^{11} \mathrm{O}$ f late, LVD D has attracted attention of the concerned people. The overall prevalence of LVDD in our OSA patients has been found to be $46.8 \%$, such a figure of prevalence being observed by others too. ${ }^{12}$ The first direct evidence that OSA might play a role in causing left ventricular dysfunction came from $\mathrm{H}$ edner and colleagues ${ }^{13}$. They reported that OSA patients had thicker LV walls and LV mass, their mass index to body surface area, was approximately $15 \%$ higher among normotensive OSA patients than in normotensive control subjects. N oda et al ${ }^{14}$ reported LV hypertrophy (LVH) in $41 \%$ of 51 OSA patients. Alchanatis et al ${ }^{5}$ also reported that LV diastolic function was impaired in 15 OSA patients with neither history of nor present systemic hypertension, compared to 11 subjects matched for age and BM I.

Contrary to available information, in our study, there is no difference between thetwo groups, with and without LVDD. The prevalence of LVDD has been found to be higher in patients of less severe OSA (mean AHI being $42.78 \pm 27.55$ per hour) which needs explanation. 
Table: 1

\begin{tabular}{|c|c|c|c|}
\hline \multicolumn{4}{|c|}{ M orphometric M esurements } \\
\hline Parameters & $\begin{array}{l}\text { LVDD } \\
(n=15)\end{array}$ & $\begin{array}{l}\text { NoLVDD } \\
(n=17)\end{array}$ & p value \\
\hline Age(years) & $52.9 \pm 7.83$ & $48.41 \pm 10.77$ & 0.19 \\
\hline$\overline{B M I}\left(\mathrm{~kg} / \mathrm{m}^{2}\right)$ & $31.10 \pm 3.83$ & $35.27 \pm 7.13$ & 0.052 \\
\hline N eck ccf (cms) & $42.10 \pm 2.92(n=14)$ & $42.2 \pm 2.82$ & 0.92 \\
\hline Waist ccf (cms) & $109.28 \pm 10(n=14)$ & $118.02 \pm 15.30$ & 0.07 \\
\hline \multicolumn{4}{|l|}{ Resting Clinical State } \\
\hline RestingPR (/min) & $80.93 \pm 17.09$ & $80.87 \pm 14.90(n=16)$ & 0.99 \\
\hline RestingSaO 2(\%) & $96.92 \pm 1.59(n=14)$ & $96.18 \pm 2.71(n=16)$ & 0.37 \\
\hline Systolic BP (mmH g) & $135.66 \pm 17.81(n=16)$ & $132.18 \pm 19.74$ & 0.6 \\
\hline Diastolic BP $(\mathrm{mm} / \mathrm{H} \mathrm{g})$ & $83.2 \pm 7.65(n=16)$ & $85.31 \pm 15.96$ & 0.64 \\
\hline \multicolumn{4}{|l|}{ CoM orbidities } \\
\hline H ypertension & $11(73.33 \%)$ & $11(64.7 \%)$ & 0.88 \\
\hline Diabetesmellitus & $4(26.66 \%)$ & $5(29.41 \%)$ & 0.82 \\
\hline IHD & $1(6.66 \%)$ & $2(11.76 \%)$ & 0.90 \\
\hline H ypothyroidism & $1(6.66 \%)$ & $3(17.64 \%)$ & 0.68 \\
\hline COPD & $1(6.66 \%)$ & $1(5.88 \%)$ & 0.52 \\
\hline Dyslepidemia & 0 & $1(5.88 \%)$ & 0.94 \\
\hline \multicolumn{4}{|l|}{ Addiction Habits } \\
\hline Smoker (past \& present) & $9(60 \%) 11$ & $(64.70 \%)$ & 0.92 \\
\hline Alcoholic (past \& present) & $9(60 \%)$ & $6(35.29 \%)$ & 0.29 \\
\hline \multicolumn{4}{|l|}{ Sleep Reated Parameters } \\
\hline $\mathrm{AHI}(/ \mathrm{hr})$ & $42.78 \pm 27.55$ & $59.83 \pm 30.12$ & 0.10 \\
\hline ESS score & $11.93 \pm 6.8$ & $15.41 \pm 5.13$ & 0.11 \\
\hline Total Sleep & $211.06 \pm 128.51$ & $202.17 \pm 71.83$ & 0.81 \\
\hline \multicolumn{4}{|l|}{ Time(mins) } \\
\hline Sleep Efficiency(\%) & $84.03 \pm 20.98$ & $91.27 \pm 5.41$ & 0.18 \\
\hline \multicolumn{4}{|l|}{ Sleep Abnormalities } \\
\hline O bstructive Apnea (/hr) & $18.88 \pm 21.35$ & $32.68 \pm 28.88$ & 0.13 \\
\hline Central Apnea(/hr) & $1.393 \pm 4.63$ & $0.35 \pm 0.70$ & 0.35 \\
\hline M ixed Apnoea(/hr) 3.5 \pm 4.77 & $4.65 \pm 7.70$ & 0.60 & \\
\hline H ypopnoea(/hr) & $19.01 \pm 14.28$ & $22.12 \pm 15.93$ & 0.56 \\
\hline SnoreT ime(mins) & $44.09 \pm 60.53$ & $19.45 \pm 14.29$ & 0.11 \\
\hline \multicolumn{4}{|l|}{ 0xygenation } \\
\hline Avg SAO 2(\%) & $92.77 \pm 4.49$ & $91.25 \pm 5.31(n=16)$ & 0.39 \\
\hline LowetSA02(\%) & $75.26 \pm 11.02$ & $67.62 \pm 12.64(n=16)$ & 0.08 \\
\hline Avg D esat (\%) & $8.51 \pm 3.18$ & $9.73 \pm 3.59(n=16)$ & 0.32 \\
\hline Sat< $<0 \%(\%)$ & $22.44 \pm 27.77$ & $29.44+29.05$ & 0.49 \\
\hline Sat <80\% (\%) & $5.82 \pm 11.15$ & $8.04 \pm 11.98$ & 0.59 \\
\hline Sat <70\% (\%) & $0.94 \pm 1.97$ & $1.78 \pm 12.51$ & 0.79 \\
\hline
\end{tabular}

However, OSA patients with LVDD were found to be slightly older than their counterparts. Duration of snoring has also been found to be more in favor of patients with LVDD, though none of the relations on either way was statistically significant. It is not possible, therefore, from the results, to associate severity of OSA with risk or prevalence of LVDD. From this point of view, the origin of LVDD in certain OSA sufferers becomes a more interesting area of research. The data and the analysis do not point to any variable in consideration being associated with the presence of diastolic dysfunction of left ventricle. Perhaps, some other factors, apparently not clear, are playing a crucial role in our patients. COPD has recently been implicated as an association of LVDD. ${ }^{15}$ We have not performed spirometry to any of these patients that could have been 
helpful to identify COPD.

There are several limitations in our study. This was a retrospectivestudy with the total number of patients being very small. There was no control group as well. Another limitation is the echocardiography was done at different places by different persons using different machines. The actual prevalence of diastolic dysfunction could be much higher if the echocardiography could have been done following a stringent protocol. We are not aware of the values of different parameters for LVDD in echocardiography. H owever, in general, left ventricular ejection fraction (LVEF), IV septal thickness, LV internal diameter, E/A ratio have been usually looked for according to those reports.

Although it is difficult to derive any inference with such a small number of patients, the higher association of LVDD in snorers needs further evaluation. The study signifies the need for a well planned prospective investigation in this area with determination of the right incidence, markers and the significance of LVDD in OSA patients.

\section{References}

1. Naughton MT. The link between obstructive sleep apnea and heart failure: underappreciated opportunity for treatment. Curr Cardiol Rep 2005;7:211-5

2. Hung J, Whitford EG, Parsons RW, Hillman DR. Association of sleep apnea with myocardial infarction in men. Lancet 1990;336:261-4.

3. Bradley TD. Right and left ventricular functional impairment and sleep apnea. Clin Chest Med 1992;13:459-79.

4. Yip G, Ho P, Woo KS, et al. Comparison of frequencies of left ventricular systolic and diastolic heart failure in Chinese living in Hong Kong. Am J Cardiol 1999;84:563-7

5. Alchanatis $\mathbf{M}$, Paradellis $\mathrm{G}$, Pini $\mathbf{H}$, et al. Left ventricular function in patients with obstructive sleep apnoea syndrome before and after treatment with nasal continuous positive airway pressure. Respiration 2000; 67:367-371.

6. Young T, Palta M, Dempsey J, Skatrud J, Weber S, Badr S. The occurrence of sleep disordered breathing among middle-aged adults. N Engl J Med. 1993;328:1230 -1235.

7. Ip MS, Lam B, Lauder I, et al. A community study of sleepdisordered breathing in middle-aged Chinese men in Hong Kong. Chest 2001;119:62-69

8. Peker Y, Hedner J, N orum J Kraiczi H, Carlson J. Increased incidence of cardiovascular disease in middle-aged men with obstructive sleep apnea: a 7-year follow-up. Am J Respir Crit Care Med 2002; 166: 159-65.

9. Hung J, Whitford EG, Parsons RW, Hillman DR. Association of sleep apnea with myocardial infarction in men. Lancet 1990;336:261-4.

10. Bradley TD. Right and left ventricular functional impairment and sleep apnea. Clin Chest M ed 1992; 13: 459-79.

11. Fung JW, Li TS, Choy DK, Yip GW et al. Severe obstructive sleep apnea is associated with left ventricular diastolic dysfunction. Chest 2002;121:422-29.

12. Sidana J, Aronow WS, Ravipati G, Stante BD, McClung JA, Belkin RN, et al. Prevalence of moderate and severe left ventricular diastolic dysfunction in obese persons with obstructive sleep apnea. Cardiology 2005;104:107-9.

13. Hedner J, Ejnell H, Caidahl K. Left ventricular hypertrophy independent of hypertension in patients with obstructive sleep apnea. J Hypertens 1990;8:941-6.

14. Noda A, O kada T, Yasuma F, Nakashima N, Yokota M. Cardiac hypertrophy in obstructive sleep apnea syndrome. Chest1995;107:1538-44.

15. Funk GC, Lang I, Schenk P, Valipour A, Hartis S, Burghuber $O C$. Left ventricular diastolic dysfunction in patients with $\mathrm{CO} P D$ in the presence and absence of elevated pulmonary arterial pressure. Chest 2008;133:1354-9. 